\title{
Apendicitis aguda perforada con diagnóstico prequirúrgico y hernia inguinal bilateral en recién nacido de pretérmino
}

\author{
Acute perforated appendicitis with pre-surgical diagnosis and bilateral inguinal hernia \\ in preterm infants
}

\section{Hernán Villalón U. a,b,c, Angélica B. Patiño ${ }^{\mathrm{d}}$, Carolina Pérez S. ${ }^{\mathrm{e}}$, Stefan Hosiasson S. ${ }^{\mathrm{a}, \mathrm{c}}$, Mauricio Pinto C. ${ }^{\mathrm{a}, \mathrm{c}}$, Patricio Varela ${ }^{\mathrm{f}}$}

\author{
aServicio de Neonatología. Clínica Las Condes. Santiago, Chile \\ bUniversidad de Chile. Santiago, Chile \\ 'Departamento de Pediatría. Universidad Finis Terrae. Santiago, Chile \\ IInterna de Pediatría. Universidad Finis Terrae. Santiago, Chile \\ eservicio de Radiología. Clínica Las Condes. Santiago, Chile \\ fUnidad Cirugía Infantil. Clínica Las Condes. Santiago, Chile
}

Recibido: 3 de diciembre de 2019; Aceptado: 28 de junio de 2020

¿Qué se sabe del tema que trata este estudio?

La apendicitis en recién nacidos es de muy baja frecuencia y alta mortalidad, entre otros factores, por la dificultad en el diagnóstico y la tardía intervención quirúrgica.
¿Qué aporta este estudio a lo ya conocido?

El mayor conocimiento, la mejoría en la resolución de los equipos de ultrasonido y especialistas entrenados, pueden contribuir de manera muy significativa a un diagnóstico prequirúrgico precoz que cambie el pronóstico.

\section{Resumen}

La apendicitis aguda neonatal es una patología de muy baja frecuencia, con escasos reportes en los últimos 30 años. La clínica e imágenes son inespecíficas, diagnosticándose la mayoría de las veces durante el procedimiento quirúrgico. Objetivo: Presentar un caso de apendicitis aguda perforada en paciente prematura, con diagnóstico prequirúrgico y resolución laparoscópica, asociada a aparición posterior de hernia inguinal. Caso Clínico: Recién nacido femenino de 34 semanas que a los 17 días de vida presentó fiebre, irritabilidad y aumento del residuo lácteo. Al examen físico destacaba distensión y sensibilidad del abdomen y tanto la radiografía como ecografía abdominales eran compatibles con apendicitis aguda perforada, por lo cual ingresó a pabellón para laparoscopía. Confirmado el diagnóstico, se realizó apendicectomía. Dos semanas después en consulta ambulatoria, se evidenció y confirmó con ecografía una hernia inguinal bilateral que se corrigió
Palabras clave: Apendicitis Aguda; Recien nacido; Abdomen Agudo

Neonatal;

Hernia Inguinal

Neonatal;

Ecografía;

Diagnóstico

Pre-Quirúrgico

Correspondencia:

Hernán Villalón

hvillalon@clinicalascondes.cl 
quirúrgicamente. Conclusión: La apendicitis aguda debe considerarse dentro de los diagnósticos diferenciales en neonatos con clínica de abdomen agudo, principalmente en prematuros. En este caso, la ecografía permitió realizar el diagnóstico pre-operatorio. La posible asociación con hernia inguinal, debiera motivar a examinar siempre conductos inguinales durante la evaluación con imágenes y en el procedimiento quirúrgico.

\section{Abstract}

Acute neonatal appendicitis is a rare pathology, with few reports in the last 30 years. Since its clinical presentation and imaging studies are non-specific, most cases are diagnosed during a surgical procedure. Objective: To describe a neonatal case of acute perforated appendicitis associated with later appearance of inguinal hernia, with pre-surgical diagnosis and treated through laparoscopy. Clinical Case: A 17-day-old preterm female newborn presented with fever, irritability, and increased milk intolerance. Physical examination showed abdominal distention, tenderness and both, abdominal Xray and ultrasound showed compatible images with acute perforated appendicitis. Once the diagnosis was confirmed, we performed an appendicectomy through laparoscopy. Two weeks later, during an outpatient visit, we observed a bilateral inguinal hernia which was confirmed by ultrasound, and then it was surgically corrected. Conclusion: Acute appendicitis should be considered within the differential diagnosis in neonates with acute abdominal symptoms, mainly in premature infants. In this case, ultrasound scan allowed us to make the pre-operative diagnosis. The possible association with inguinal hernias should motivate to examine inguinal ducts during imaging assessment and surgical procedure.

\section{Keywords:}

Acute Appendicitis;

Newborn;

Premature;

Acute Neonatal

Abdomen;

Neonatal Inguinal

Hernia;

Ultrasound; Pre-Surgical Diagnosis

\section{Introducción}

La apendicitis neonatal es una patología muy poco frecuente, con menos de 50 casos reportados en los últimos $30 \mathrm{años}^{1-3}$. En la mayoría de los casos reportados, la apendicits neonatal estuvo asociada con alguna otra patología: enfermedad de Hirschsprung, ileo meconial y hernia inguinal uni o bilateral, entre otras $^{4}$. Los factores de riesgo de esta condición se relacionan a condiciones que generan hipoxia-isquemia ${ }^{1}$, lo que podría explicar la fisiopatología detrás de este cuadro.

Los síntomas suelen ser inespecíficos, predominando la distensión abdominal y vómitos, y en la mayoría de los casos, se presentan síntomas propios de una enterocolitis necrosante ${ }^{2}$. La ecografía puede resultar útil para el diagnóstico, pero tiene limitaciones $^{3}$. En general, es una patología que evoluciona rápido a la perforación y peritonitis ${ }^{2}$, ya que su diagnóstico suele ser tardío y la mayoría de las veces un hallazgo quirúrgico en el contexto de un cuadro compatible con abdomen agudo. El tratamiento corresponde a la resección quirúrgica ${ }^{1}$. Por la escasa frecuencia de este diagnóstico y su importancia en el recién nacido, se presenta este caso clínico de apendicitis aguda perforada en una paciente prematura, con diagnóstico radiológico pre-quirúrgico y resolución laparoscópica, asociada a aparición posterior de una hernia inguinal.

\section{Caso Clínico}

Recién nacido femenino, prematura de 34 semanas, gemela I, hija de madre primigesta sana. Su madre tuvo trabajo de parto prematuro refractario a Atosiban, por lo que se realizó cesárea con extracción cefálica no complicada. Nace con peso $2.075 \mathrm{~g}$, Talla $43,5 \mathrm{~cm}$, puntaje Apgar 8-8, y su examen físico fue normal. Fue ingresada a Unidad de Paciente Crítico Neonatal, donde se tomó una muestra para hemocultivos y posteriormente inició antibioterapia biasociada. Dentro de primeras horas presentó dificultad respiratoria, que fue manejada con CPAP por $8 \mathrm{~h}$ y posteriormente oxígeno por $48 \mathrm{~h}$. Inició alimentación a las $24 \mathrm{~h}$ de vida, con buena tolerancia al aumento progresivo. Al 3er día de vida se decidió suspender los antibióticos dado que tenía cultivos negativos y dos controles de hemograma y proteína $\mathrm{C}$ reactiva dentro de lo normal.

A los 17 días de vida presentó fiebre, irritabilidad y residuo gástrico bilioso. En el examen físico destacó un abdomen blando, pero distendido y sensible, con ruidos hidro-aéreos presentes. La radiografía simple de abdomen mostró distribución patológica del aire intestinal, con escasa representación en flanco y fosa ilíaca derecha y presencia de burbujas aéreas aisladas confluentes sospechosas de aire extraintestinal (figura 1).

En la ecografía abdominal se observó significativo menor contenido aéreo en asas intestinales a derecha, con peristaltismo disminuido y paredes engrosadas, 


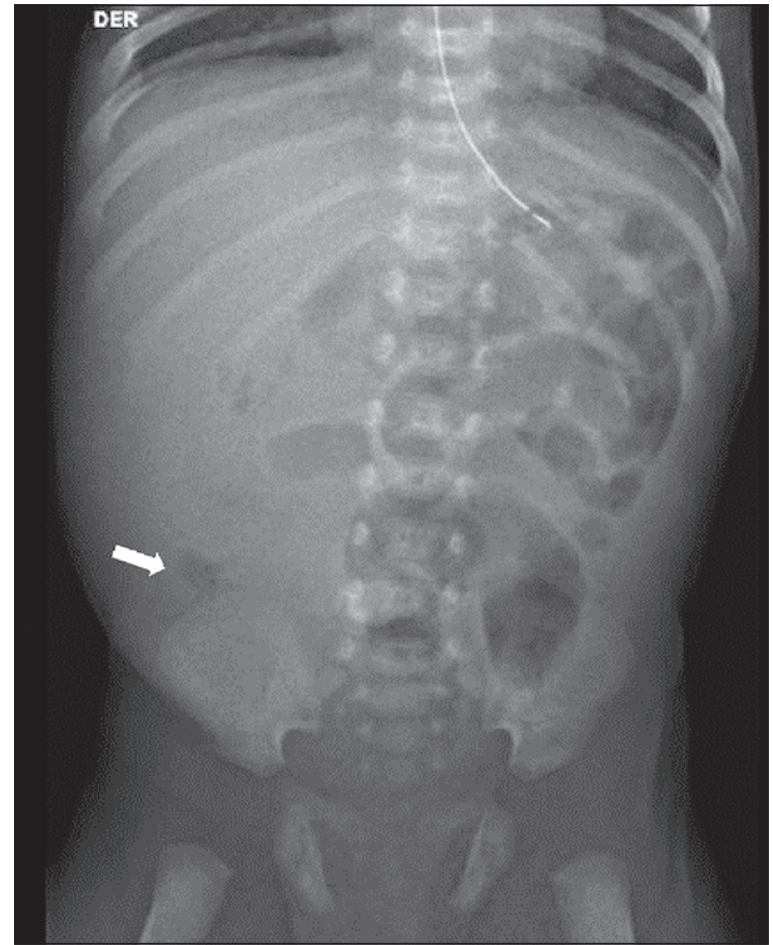

Figura 1. Radiografía simple de abdomen. Muestra distribución aérea patológica y burbujas confluentes sugerentes de aire extraintestinal (señalado con flecha).

acompañado de una mayor ecogenicidad del plano adiposo lateral y posterior en flanco y fosa ilíaca derecha. Se observó líquido libre interasas escaso de aspecto ecogénico en fosa ilíaca derecha (figura 2a). Se logró visualización parcial de apéndice cecal, siguiendo curso retrocecal, engrosado, con diámetro de $6 \mathrm{~mm}$, terminando en área mal delimitada de tejido hipoecogénico heterogéneo y burbujas aéreas confluentes extraluminales, sugerente de perforación (figura $2 b$ ). Con éstos hallazgos se planteó diagnóstico ecográfico de proceso inflamatorio en flanco-fosa ilíaca derecha, probable apendicitis aguda complicada. No se describieron los ductos inguinales.

Dentro de los examenes de laboratorio destaca un hemograma con leucocitosis $\left(24.700\right.$ por $\left.\mathrm{mm}^{3}\right)$ y Proteína C Reactiva $54 \mathrm{mg} / \mathrm{l}$. Inició tratamiento antibiótico triasociado (Ampicilina, Amikacina y Metronidazol). La exploración laparoscópica demostró apéndice con perforación apical y líquido libre purulento. Se realizó apendicetomía y lavado de cavidad. No se realizó exploración de conductos inguinales.

La biopsia confirmó apendicitis aguda flegmonosa perforada, sin malignidad. En cultivo peritoneal se obtuvo Enterococo sensible a Ampicilina. Completó 7 días con antibióticos, presentando una favorable evolución, que permitió el alta a los diez días.

Dos semanas después, en control médico ambula-

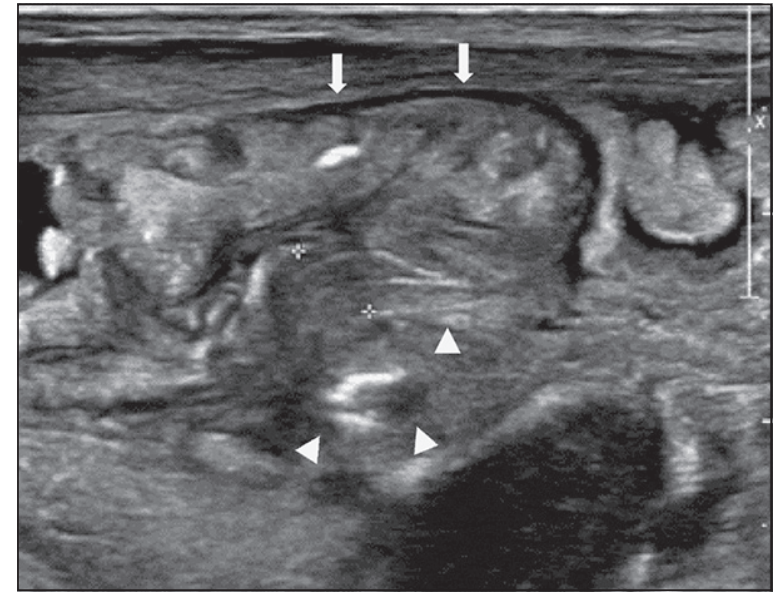

Figura 2a. Ecotomografía abdominal: Muestra engrosamiento de asas intestinales y mayor ecogenicidad en tejido adiposo de flanco y fosa ilíaca derecha.

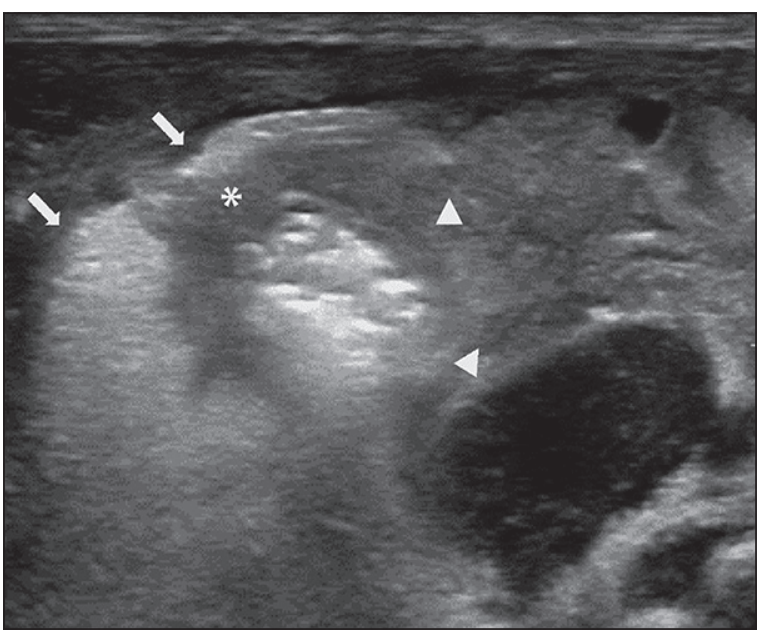

Figura 2b. Ecotomografía abdominal: Muestra apéndice retrocecal engrosado con ápice en área hipoecogénica mal delimitada, y burbujas confluentes extraluminales, sugerentes de perforación.

torio se evidenció aumento de volumen inguinal no reductible. La ecografía mostró una hernia bilateral con deslizamiento de ovario a izquierda. Se realizó corrección quirúrgica, sin presentar otras complicaciones.

\section{Discusión}

La apendicitis en el período neonatal es un cuadro raro cuya incidencia estimada es $0,04-0,02 \%^{2}$ y que conlleva morbimortalidad aún muy alta ${ }^{6}$. El $75 \%$ de los casos corresponde a hombres, y un $52 \%$ son nacidos de pretérmino ${ }^{7}$.

Diversos reportes han destacado la similitud clínica con enterocolitis necrosante, por lo que se ha plantea- 
do tanto una posible asociación entre ambas condiciones, como que la apendicitis en el recién nacido podría estar en el contexto de la ella ${ }^{2,5,6,8}$. Otras condiciones asociadas que se han observado son la enfermedad de Hirschsprung, fibrosis quística, íleo meconial, hernia inguinal uni o bilateral, o infección por citomegalovirus ${ }^{3,4}$, aunque en algunas series reportadas no se ha demostrado la asociación con enfermedad de Hirschprung ni fibrosis quística9. Por la baja incidencia de esta condición, las series reportadas cuentan con pocos casos.

La sintomatología es inespecífica. La presentación más común es la distensión abdominal, la que se puede acompañar de sensibilidad, irritabilidad, inestabilidad térmica, rechazo alimentario, dificultad respiratoria y un contexto clínico séptico ${ }^{2,3}$ con una rápida evolución que con frecuencia condiciona un mal pronóstico vita $^{3,4,6,7,10}$, alcanzando en algunos reportes hasta un $100 \%$ de letalidad ${ }^{11}$.

Diversos factores, tanto anatómicos como ambientales, explicarían la baja incidencia en recién nacidos. El apéndice es más corto, con una base más amplia en forma de embudo y una posición más recostada. También contribuyen la dieta láctea sin sólidos, la falta de coprolitos y la ausencia de hiperplasia linfoide ${ }^{3,12}$. Entre los factores causales, un ambiente de isquemia, y otras condiciones que promueven esta situación, como la hipoxia neonatal, preeclampsia, cateterismo umbilical, prematuridad, insuficiencia vascular asociada a anomalías cardíacas y estados de bajo flujo sanguíneo, podrían favorecer su desarrollo ${ }^{1}$.

En la actualidad el manejo quirúrgico es mayoritariamente laparoscópico ${ }^{14}$ y tiene objetivos tanto diagnóstico como terapéutico. La precocidad del diagnóstico tiene gran importancia en el pronóstico, tanto en las complicaciones sépticas como en la sobrevida, considerando que por las características clínicas el diagnóstico suele ser tardío ${ }^{1,4,5,6,14,15}$. Algunas propuestas de tratamiento conservador, con antibióticos y sin cirugía, hasta hoy, no cuentan con evidencia en recién nacidos ${ }^{16}$.

El estudio a través de imágenes ha contribuido de manera significativa al diagnóstico temprano, aunque hasta hace pocos años no se había reportado casos en que éste fuera preoperatorio ${ }^{1,7,13}$. Publicaciones recientes han enfatizado en el mayor rendimiento de la ecografía en el diagnóstico y de complicaciones en las apendicitis, cuando son realizadas por radiólogos pediátricos. Sin embargo, y a pesar de los avances, los reportes son escasos en recién nacidos ${ }^{17,18}$. En un reporte de cuatro casos de recién nacidos con diagnóstico ecográfico pre-operatorio de apendicitis, los hallazgos descritos fueron dilatación ileocecal, engrosamiento de la pared intestinal y ciego, líquido localizado y encapsulado en el cuadrante inferior derecho ${ }^{19}$. Aunque la ecografía tiene limitaciones, ha demostrado ser más efectivo que las radiografías simples para evidenciar líquido libre intraabdominal, evaluar el grosor de la pared intestinal y la perfusión de tubo digestivo ${ }^{13}$. Las principales limitaciones de la ecografía se relacionan con el método, ya que al ser un procedimiento operador-dependiente su rendimiento es mayor en manos experimentadas, y en casos de sospecha clínica tardía del diagnóstico, se hace aún más dificil la diferenciación con otros procesos inflamatorios, como la enterocolitis necrotizante complicada ${ }^{18}$. En general el diagnóstico preoperatorio es infrecuente ${ }^{7}$. En el caso reportado, el diagnóstico se hizo antes del procedimiento quirúrgico laparoscópico, gracias a los hallazgos ecográficos.

Otros desafíos en el diagnóstico radiológico es sospechar la inflamación apendicular cuando se encuentra dentro del conducto inguinal (Hernia de Amyand) ${ }^{20}$, así como otras complicaciones, como es el caso de la apendicitis en el contexto de una enterocolitis necrotizante ${ }^{5,6,21}$, hernias atascadas bajo las cuales subyace una apendicitis complicada ${ }^{10}$, cuadros que se presentan como torsión testicular u ovárica, en las cuales hay un apéndice inflamado y perforado ${ }^{22,23}$. Hacer el diagnóstico en forma oportuna permite orientar de mejor manera las estrategias quirúrgicas.

Si bien la mortalidad de la apendicitis ha disminuido de $78 \%$ entre los años 1901-1975 a 28\% en 19852003, todavía es demasiado alta debido al retraso en el diagnóstico y tratamiento, en un grupo de alta vulnerabilidad como es el recién nacido ${ }^{24}$.

Estudios retrospectivos han observado asociaciones de hernia inguinal y apendicitis con manifestaciones inguinoescrotales, debido a desplazamiento dentro del conducto inguinal del apéndice mismo y/o productos de la inflamación ${ }^{25}$. En el caso expuesto, no se observó esta forma de presentación, ya que la hernia se manifestó dos semanas más tarde. Sin embargo, la condición anatómica estaba presente. Se podría sugerir que al sospechar un fenómeno inflamatorio apendicular en recién nacidos, se examinen detenidamente los conductos tanto con ecografía, como en el momento de la exploración laparoscópica.

\section{Conclusión}

Aunque muy poco frecuente, la apendicitis debe incluirse entre los diagnósticos diferenciales de los trastornos abdominales agudos en recién nacidos y prematuros, considerando la alta morbimortalidad. Los radiólogos pediátricos entrenados podrían optimizar el diagnóstico precoz, lo que permite un manejo oportuno. La hernia inguinal debe incluirse en las condiciones a pesquizar, considerando la asociación entre apendicitis neonatal y la presencia de hernia inguinal. 


\section{Responsabilidades Éticas}

Protección de personas y animales: Los autores declaran que los procedimientos seguidos se conformaron a las normas éticas del comité de experimentación humana responsable y de acuerdo con la Asociación Médica Mundial y la Declaración de Helsinki.

Confidencialidad de los datos: Los autores declaran que han seguido los protocolos de su centro de trabajo sobre la publicación de datos de pacientes.
Derecho a la privacidad y consentimiento informado: Los autores han obtenido el consentimiento informado de los pacientes y/o sujetos referidos en el artículo. Este documento obra en poder del autor de correspondencia.

\section{Conflicto de intereses}

Los autores declaran no tener conflicto de intereses.

\section{Referencias}

1. Secco IL, Costa T, Moraes ELL, Freire MHS, Danski MTR, Cavalcante RPGV. Neonatal appendicitis: a survival case study. Rev Bras Enferm. 2017;70(6):1296-1300. doi: 10.1590/00347167-2016-0610

2. Haider F, Ayoub B, Al Kooheji M, Al Juffairi M, Al-Shaikh S. Perforated acute appendicitis with no peritonitis in a premature baby: a case report. J Med Case Reports. 2017;11:125. https://doi. org/10.1186/s13256-017-1289-0.

3. Mammou S, Ayadi I, Hamida E Marrakchi Z. Acute neonatal appendicitis in a preterm. African Journal of Paediatric Surgery. 2015;12(4):294.

4. Casal-Beloy I, García-González M, García-Novoa M. Appendicitis, an unusual cause of acute abdomen in neonatal patients. A case report. Anales del Sistema Sanitario de Navarra. 2018;412.

5. Tumen A, Chotai PN, Williams JM, et al. Neonatal Perforated Appendicitis Masquerading as Necrotizing Enterocolitis. J Neonatal Surg. 2017;6(2):39. doi: 10.21699/jns.v6i2.482.

6. Tumen A, Chotai PN, Williams JM, Myers-Webb A, Krishnan R, Eubanks Iii JW. Neonatal Perforated Appendicitis Attributed to Localized Necrotizing Enterocolitis of the Appendix: A Review. J Neonatal Surg. 2017;6(3):60. doi: 10.21699/jns.v6i3.535.

7. Romero Rojas N, Sánchez García L. Apendicitis aguda neonatal: reporte de un caso. Anales de la Facultad de Medicina. 2016;77(4):421.

8. Pressman A, Kawar B, Abend M, Steiner Z, Mogilner G. Acute Perforated Neonatal Appendicitis Associated with Chorioamnionitis. Eur J Pediatr Surg
2001;11(3):204-6. doi: 10.1055/s-200115481.

9. Gregorio-Hernández R, Pérez-Pérez A, Arriaga-Redondo M, Simal I, SánchezLuna M. Apendicitis abdominal neonatal. Experiencia en un hospital terciario y revisión de la literatura. Acta Pediatr Esp. 2017;75(7-8): e138-e140.

10. Fascetti-Leon F, Sherwood W. Neonatal Appendicitis and Incarcerated Inguinal Hernia: Case Report and Review of the Literature. J Indian Assoc Pediatr Surg. 2017;22 (4):248-50. doi: 10.4103/jiaps. JIAPS_226_16.

11. Amin $\mathrm{M}, \mathrm{Al}$ Jubouri S. Neonatal appendicitis with perforation: a case report. J Ped Surg Case Reports 2014;2:353-4.

12. Marzuillo P, Germani C, Krauss B, Barbi E. Appendicitis in children less than five years old: A challenge for the general practitioner. World Journal of Clinical Pediatrics. 2015 4(2):19-24.

13. Schwartz KL, Gilad E, Sigalet D, Yu W, Wong AL. Neonatal acute appendicitis: a proposed algorithm for timely diagnosis. J Pediatr Surg. 2011;46(11):2060-4. doi: 10.1016/j.jpedsurg.2011.07.018.

14. Stringer MD. Acute appendicitis. J Paediatr Child Health. 2017;53(11):10716. doi: 10.1111/jpc.13737.

15. Semerci SY, Kurnaz D, Babayigit A, et al. Neonatal Appendicitis as a Rare Cause of Intestinal Perforation in a Preterm Neonate. J Coll Physicians Surg Pak. 2017;27(3):S38-S40. doi: 248.

16. Yamanaka S, Skarsgard ED, Goldman RD. Conservative therapy for appendicitis in children. Can Fam Physician. 2018;64(8):574-6

17. Rawolle T, Reismann M, Minderjahn MI, et al. Sonographic differentiation of complicated from uncomplicated appendicitis. Br J Radiol.
2019;92(1099):20190102. doi: 10.1259/ bjr.20190102.

18. Reddan T, Corness J, Harden F, Mengersen K. Improving the value of ultrasound in children with suspected appendicitis: a prospective study integrating secondary sonographic signs. Ultrasonography. 2019;38(1):67-75. doi: 10.14366/usg.17062.

19. Si SY, Guo YY, Mu JF, Yan CY. The sonographic features of neonatal appendicitis: A case report. Medicine (Baltimore). 2017;96(45):e8170. doi: 10.1097/MD.0000000000008170.

20. Dhanasekarapandian V, Shanmugam V, Jagannathan M. Amyand's Hernia, Appendicitis, and Undescended Testis: The Amyand's Triad. J Indian Assoc Pediatr Surg. 2018;23(3):169-170. doi: 10.4103/jiaps.JIAPS_174_17.

21. Feldens L, Souza JCK, Fraga JC. Perforation of the cecal appendix in the neonatal period. J Pediatr (Rio J). 2018;94(5):567-8. doi: 10.1016/j. jped.2018.07.002.

22. Erginel B, Soysal FG, Celik A, Salman T. Neonatal perforated appendicitis in incarcerated inguinal hernia in the differential diagnosis of testis torsion. Pediatr Int. 2017;59(7):831-832. doi: $10.1111 /$ ped.13297.

23. Perger L, Muensterer OJ. Laparoscopic appendectomy for torsed appendix presenting as an acute abdomen in an infant female. JSLS. 2011;15(4):565-7. doi: 10.4293/108680811X13176785204634.

24. Raveenthiran V. Neonatal Appendicitis (Part 1): A Review of 52 cases with abdominal manifestation. J Neonat Surg. 2015;4(1):4

25. Raveenthiran V. Neonatal Appendicitis (Part 2): A Review of 24 cases with inguinoscrotal manifestation. J Neonat Surg. 2015;4(2):15. 